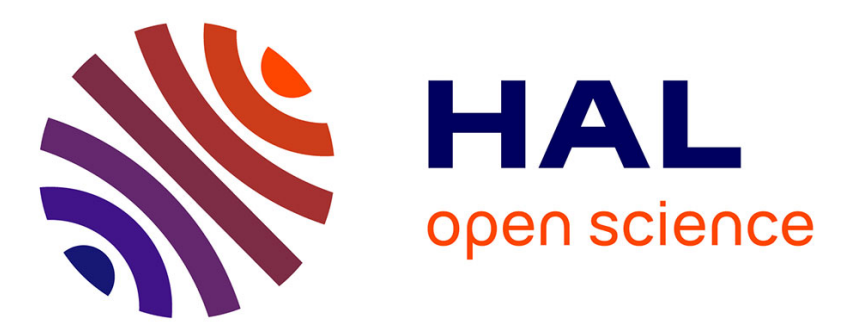

\title{
La transmission d'énergie par couplage résonnant entre une onde libre et une onde guidée
}

\author{
J. Perdijon
}

\section{To cite this version:}

J. Perdijon. La transmission d'énergie par couplage résonnant entre une onde libre et une onde guidée. Revue de Physique Appliquée, 1985, 20 (3), pp.173-181. 10.1051/rphysap:01985002003017300 . jpa00245320

\section{HAL Id: jpa-00245320 https://hal.science/jpa-00245320}

Submitted on 1 Jan 1985

HAL is a multi-disciplinary open access archive for the deposit and dissemination of scientific research documents, whether they are published or not. The documents may come from teaching and research institutions in France or abroad, or from public or private research centers.
L'archive ouverte pluridisciplinaire HAL, est destinée au dépôt et à la diffusion de documents scientifiques de niveau recherche, publiés ou non, émanant des établissements d'enseignement et de recherche français ou étrangers, des laboratoires publics ou privés. 


\title{
La transmission d'énergie par couplage résonnant entre une onde libre et une onde guidée
}

\author{
J. Perdijon \\ COGEMA, Centre d'Etudes Nucléaires, 85 X, 38041 Grenoble Cedex, France
}

(Reçu le 25 juin 1984, révisé le 16 novembre, accepté le 26 novembre 1984)

\begin{abstract}
Résumé. - On a cherché à modéliser la répartition de l'énergie le long d'un guide, plan ou tubulaire, au cours du couplage résonnant entre une onde libre et une onde guidée. Ce modèle a ensuite été comparé aux mesures effectuées autour d'une plaque guidant des ondes de Lamb. Il a enfin été appliqué à des contrôles ultrasonores de plaques ou de tubes par échographie.
\end{abstract}

\begin{abstract}
It is intended to give a model of the energy distribution along a plane or a tubular waveguide, during the resonant coupling of a bounded beam with the guide. This model has then been compared with measurements made around a plate guiding Lamb waves. Finally, it has been applied to ultrasonic testing of plates and tubes by echography.
\end{abstract}

\section{Introduction.}

La génération d'une onde guidée à l'intérieur d'un certain guide nécessite de coupler ce guide avec un émetteur extérieur au guide. Plusieurs méthodes de couplage ont été proposées. Lorsqu'il s'agit par exemple de lumière émise par un laser, on peut disposer la source à une extrémité du guide (cas d'une fibre), ou bien la coupler avec l'une des faces du guide (cas d'un film) au moyen d'un prisme ou d'un réseau [1]. Lorsqu'il s'agit d'ultrasons émis par une pastille piézoélectrique, le couplage avec un guide plat se fait soit par excitation directe sur une face, soit encore par l'intermédiaire d'un prisme ou d'un réseau [2]; dans le cas de la méthode par immersion en incidence oblique, le prisme est alors liquide. Cependant, on cherche généralement à engendrer préférentiellement dans le guide un seul mode de l'onde guidée. Il faut donc synchroniser les ondes incidentes sur le guide avec le mode qu'on veut guider dans le guide; on dit alors que le couplage est résonnant et c'est précisément ce que permet d'obtenir un prisme ou un réseau. Nous ne nous intéresserons ici qu'à ce type de couplage.

Dans tout ce qui suit, les ondes pourront aussi bien être élastiques qu'électromagnétiques; néanmoins, les vérifications expérimentales seront menées avec des ultrasons. Ces ondes seront émises par une source plane et cohérente; on envisagera le cas d'une onde continue ainsi que celui d'une impulsion, et on considérera dans les applications que le profil de la distribution de l'amplitude dans le faisceau est rectangulaire ou gaussien. Le guide sera une plaque, plane ou tubulaire, dont l'épaisseur permettra de guider un ou plusieurs modes; la configuration tubulaire présente l'intérêt de fermer le guide sur lui-même. On envisagera le cas de la présence d'un réflecteur plus ou moins parfait à l'intérieur du guide. Enfin, le coupleur permettra de vérifier la condition de synchronisation entre faisceau incident et onde guidée sur une certaine longueur du guide; l'étude sera menée en considérant un prisme mais elle pourrait être étendue au cas d'un réseau.

Du point de vue énergétique, on peut schématiser le système étudié de la façon suivante. Durant le couplage de la source avec le guide, cinq types d'onde sont principalement en présence le long du guide (Fig. 1a). Dans le milieu I situé du côté du coupleur, il y a l'onde incidente (1), l'onde réfléchie (2) et l'onde rayonnée par le guide (3); on sait que, dans les conditions habituelles de couplage, les ondes (2) et (3) sont en opposition de phase $[3,4]$. Dans le guide, il y a l'onde guidée (4). Et, dans le milieu II situé de l'autre côté du guide, il peut exister une seconde onde rayonnée (5) si l'indice de réfraction de ce milieu est supérieur à celui du guide $\left({ }^{1}\right)$. Ce schéma ne tiẹt pas compte de la

( $\left.{ }^{1}\right)$ Dans les sondages sous-marins, les milieux I et II sont identiques; en contrôle non-destructif et en optique, le milieu II est généralement d'indice inférieur à celui du guide. 


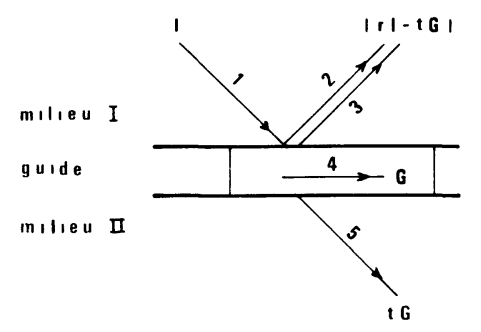

a

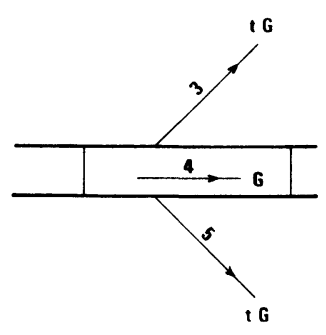

b

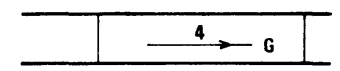

C

Fig. 1. - Répartition des amplitudes : a) durant le couplage du guide avec la source, b) après désaccouplement de la source, c) après désaccouplement des milieux environnants.

[Amplitude distribution : a) during the coupling of the guide with the source, b) after discoupling from the source, c) after discoupling from surrounding media.]

présence éventuelle d'autres types d'onde (ondes libres longitudinales ou transversales, autres modes d'onde guidée), qui sont minimisés au moment de la résonance, ainsi que des ondes évanescentes, dont le rôle est négligeable dans un bilan énergétique. En dehors de la zone de couplage entre la source et le guide, il ne reste plus en présence que les ondes (3), (4) et (5) (Fig. 1b); l'onde guidée s'amortit alors principalement par son rayonnement dans les milieux I et II. Cependant, si on désaccouple en plus le guide de ces milieux, il ne reste que l'onde guidée (Fig. 1c), qui s'amortit lentement sous l'effet de la viscosité du guide. Lors des expériences, seules les ondes libres (1), (2), (3) et (5) sont accessibles pour une mesure de leur amplitude.

Nous allons étudier la répartition de l'énergie entre ces différents types d'onde, dans plusieurs configurations possibles du système émetteur-coupleur-guide, mais il n'est pas question de refaire ici une étude théorique à partir des propriétés (élastiques ou électromagnétiques) des milieux [5-7]. Le principe de calcul utilisé sera celui de la variation du bilan énergétique le long du guide. On cherchera donc une équation différentielle exprimant cette variation, en prenant comme élément de guide pour calculer les bilans successifs une longueur égale à la longueur d'onde guidée; en effet, puisqu'il s'agit d'un couplage résonnant, toutes les ondes en présence sont synchrones et on peut raisonner sur les énergies moyennes échangées au cours d'une période. Cependant, on calculera de préférence les amplitudes dans les applications numériques, car les mesures expérimentales en ultrasons conduisent à des tensions qui sont proportionnelles aux pressions acoustiques, donc aux amplitudes et non aux énergies [8].

\section{Onde continue et guide plat sans réflecteur.}

2.1 CALCUl DE L'ÉNERGIE GUIDÉE. - Reprenons le schéma de la figure 1a et considérons un élément de guide de longueur $\lambda$, en appelant $\lambda$ la longueur de l'onde guidée. Soit $I$ l'amplitude de l'onde incidente (1) sur cet élément; l'énergie correspondante, reçue par cet élément, est proportionnelle à $I^{2}$ et, en appelant $k$ le coefficient de proportionnalité, l'énergie reçue par un élément de longueur $\mathrm{d} x$ est égale à $k I^{2} \mathrm{~d} x / \lambda$. Si $r$ est le coefficient de réflexion de l'onde incidente sur le guide, l'amplitude de l'onde réfléchie (2) est $r I$. Soit $G$ l'amplitude de l'onde guidée dans l'élément considéré; en appelant $k^{\prime}$ le coefficient de proportionnalité, l'énergie guidée est égale à $k^{\prime} G^{2}$. D'après les relations de Stokes, les coefficients de réflexion de part et d'autre du dioptre limitant le guide sont égaux et opposés et le coefficient de transmission $t$ du guide vers le milieu I est tel que $k t^{2}=k^{\prime}\left(1-r^{2}\right)$. En supposant que les milieux I et II sont identiques, l'amplitude des ondes rayonnées (3) et (5) est donc $t G$; cependant, par suite de l'interférence entre les ondes (2) et (3), en opposition de phase, l'onde retournant vers le milieu I a pour amplitude $|r I-t G|$. Puisque les milieux I et II sont identiques, on a le même coefficient de proportionnalité $k$ et l'énergie rendue au milieu I par un élément de longueur $\mathrm{d} x$ est égale à $k(r I-t G)^{2} \mathrm{~d} x / \lambda$, celle rendue au milieu II par le même élément est égale à $k t^{2} G^{2} \mathrm{~d} x / \lambda$. Enfin l'énergie absorbée dans cet élément de guide par sa viscosité est égale à $k^{\prime} \alpha G^{2} \mathrm{~d} x$, en appelant $\alpha$ le coefficient d'absorption.

Ecrivons maintenant le bilan énergétique pour l'élément $\mathrm{d} x$. La variation de l'énergie guidée est égale à la différence entre l'énergie reçue (de (1)) et les énergies rendues (au milieu I par (2) et (3) et au milieu II par (5)) et absorbée, soit :

$$
\begin{array}{r}
k^{\prime} \mathrm{d}\left(G^{2}\right)=k\left[I^{2}-(r I-t G)^{2}-t^{2} G^{2}\right] \mathrm{d} x / \lambda- \\
-k^{\prime} \alpha G^{2} \mathrm{~d} x
\end{array}
$$

ou, en posant $G^{2}=y$ et $k^{\prime} \lambda / k=\Lambda$ :

$$
\Lambda y^{\prime}=\left(1-r^{2}\right) I^{2}+2 r t I y^{1 / 2}-\left(2 t^{2}+\alpha \Lambda\right) y .
$$

En l'absence de couplage avec le milieu II (milieu II d'indice inférieur à celui du guide), (2) devient :

$$
\Lambda y^{\prime}=\left(1-r^{2}\right) I^{2}+2 r t I y^{1 / 2}-\left(t^{2}+\alpha \Lambda\right) y .
$$

En l'absence de couplage avec la source $(I=0)$, (2) devient (Fig. 1b) :

$$
\Lambda y^{\prime}=-\left(2 t^{2}+\alpha \Lambda\right) y
$$


et (3) devient :

$$
\Lambda y^{\prime}=-\left(t^{2}+\alpha \Lambda\right) y .
$$

En l'absence de couplage avec les deux milieux (confinement), (2) devient (Fig. 1c) :

$$
y^{\prime}=-\alpha y .
$$

Pendant toute la durée du couplage entre la source et le guide, l'équation différentielle est donc du type (2) ou (3); elle n'est pas intégrable analytiquement. Considérons d'abord le cas d'un faisceau incident dont l'amplitude est constante et égale à $I$ à partir de $x=0$. Lorsque $x$ est petit, c'est-à-dire au début du couplage, l'énergie de l'onde guidée croît pratiquement linéairement, avec une pente égale à $k\left(1-r^{2}\right) I^{2} / \lambda$. Lorsque $x$ tend vers un grand nombre de longueurs d'onde, on observe une saturation, comme avec l'équation obtenue par Tien et Ulrich [3]. Si on pose $A=G / I, A$ représente un coefficient d'amplification qui tend vers :

$A_{\infty}=\left\{r t+\left[r^{2} t^{2}+\left(1-r^{2}\right)\left(p t^{2}+\alpha \Lambda\right)\right]^{1 / 2}\right\} /\left(p t^{2}+\alpha \Lambda\right)$,

en désignant par $p$ le nombre de faces du guide couplées avec les milieux extérieurs; pour $p=1$ et $\alpha \simeq 0$, on a :

$$
A_{\infty}=(1+r) / t
$$

qui tend vers $2 / t$ lorsque $r$ est voisin de 1 . Cependant, cette saturation n'est atteinte que si le couplage entre la source et le guide est réalisé sur une longueur suffisante. On peut aussi définir à partir de $A$ un rendement, rapport entre l'énergie guidée et celle qu'il a fallu fournir au guide :

$$
\eta=A^{2} \Lambda / x
$$

La résolution numérique de l'équation différentielle (2) ou (3), par exemple au moyen de la méthode de RungeKutta [9], permet de calculer l'amplitude guidée $G$ en tout point du guide, même lorsque $I$ est une fonction (en particulier gaussienne) de $x$; on en déduit les amplitudes $|r I-t G|$ et $t G$ des ondes retournant vers les milieux I et II.

Lorsque la source n'est plus couplée au guide, l'intégration de (4), (5) ou (6) montre que l'énergie guidée décroît exponentiellement. Si on appelle $s$ la longueur de couplage et $G_{\mathrm{m}}$ l'amplitude obtenue à la fin du couplage, on a :

$$
G^{2}=G_{\mathrm{m}}^{2} \exp [-\beta(x-s)],
$$

en posant $\beta=p t^{2} / \Lambda+\alpha$.

2.2 Mesures en ondes de Lamb. - On a tenté de mesurer les amplitudes des différentes ondes libres en présence le long du guide dans le cas où ces ondes sont ultrasonores [2]. Pour ce faire, on a utilisé le dispositif suivant (Fig. 2), dont le principe a déjà été décrit [10]. Une plaque $(P)$ en acier inoxydable, de grandes dimensions et d'épaisseur $1 \mathrm{~mm}$, est suspendue verticalement suivant un plan $x \mathrm{O} z$ dans une cuve d'eau. Un plateau, disposé au-dessus de la cuve, comporte deux bras orientables en azimut autour d'axes verticaux situés dans le plan $x \mathrm{O} z$. Ces bras sont équipés chacun d'un porte-traducteur; les axes des deux portetraducteur sont situés dans le même plan horizontal $x \mathrm{O} y$ et l'un des deux bras peut être déplacé parallèlement au plan $x \mathrm{O} z$.

L'émetteur (E) est monté dans le porte-traducteur du bras fixe. Il est du type NDC CNG 4 DA/1 ; c'est un traducteur piézoélectrique plan de diamètre $20 \mathrm{~mm}$, de fréquence $4 \mathrm{MHz}$ et moyennement amorti. Le parcours préliminaire dans l'eau est de $150 \mathrm{~mm}$ et l'angle d'incidence est réglé au moyen du goniomètre lié au bras. L'émetteur est excité par un générateur, du type Schlumberger 4432, en ondes sinusoïdales de fréquence variable autour de la fréquence propre de l'émetteur; la tension d'excitation est alors de l'ordre de $\pm 1,5 \mathrm{~V}$. Quant à la sonde $(\mathrm{S})$ qui doit analyser les faisceaux, elle est montée dans le portetraducteur du bras mobile. Il s'agit d'un traducteur piézoélectrique, du type CGR $484 \mathrm{~A} / \mathrm{EL}$, de diamètre $10 \mathrm{~mm}$, de fréquence $5 \mathrm{MHz}$, avec une focalisation ponctuelle à $50 \mathrm{~mm}$. Cette sonde est disposée de façon que le foyer soit sur la plaque et son angle d'incidence, réglé au moyen du goniomètre lié au bras, est le même que celui de l'émetteur; elle est connectée à un oscilloscope du type Tektronix 465.

Le mode opératoire est le suivant [10]. L'angle d'incidence est choisi d'après les courbes donnant la vitesse de phase des ondes de Lamb en fonction du produit fréquence $\times$ épaisseur [11]. On dispose la sonde du même côté de la plaque que l'émetteur mais avec un angle d'incidence opposé à celui de l'émetteur

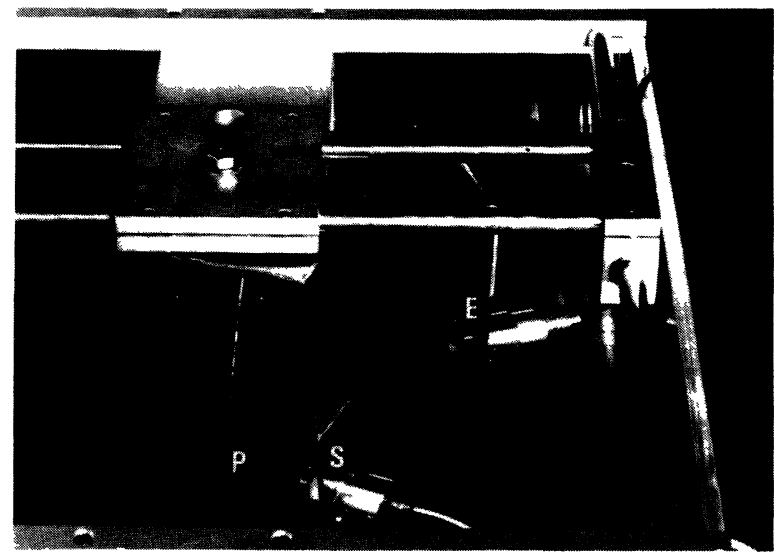

Fig. 2. - Dispositif pour mesurer la répartition de l'énergie ultrasonore autour d'une plaque $(\mathrm{P}=$ plaque, $\mathrm{E}=$ émetteur, $\mathrm{S}=$ sonde).

[Device for measuring the distribution of the ultrasonic energy around a plate $(P=$ plate, $E=$ transmitter, $S=$ receiver).] 


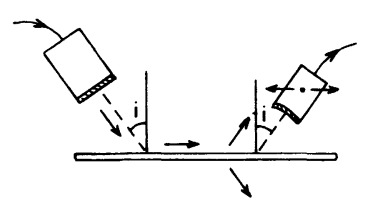

a

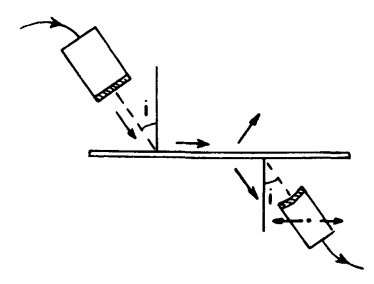

b

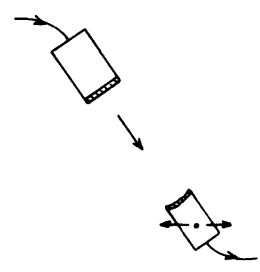

c

Fig. 3. - Principe de la mesure des amplitudes dans les faisceaux ultrasonores autour d'une plaque : a) faisceau retournant au milieu I, b) faisceau retournant au milieu II, c) faisceau incident.

[Principle of the amplitude measurements in the ultrasonic beams around a plate : a) beam to medium I, b) beam to medium II, c) incident beam.]

(Fig. 3a). Puis on déplace la sonde de façon qu'elle analyse une zone située à la limite mais en dehors du faisceau incident; on optimise alors le signal mesuré par la sonde en faisant varier la fréquence $f$ du générateur : quand une résonance est observée, on considère que la plaque est excitée en ondes de Lamb. Puis on rapproche la sonde de l'émetteur jusqu'à obtenir un minimum du signal analysé; on minimise alors ce signal en faisant très légèrement varier $f$ : l'interférence entre les ondes (2) et (3) montre que la génération d'ondes de Lamb est bien favorisée. Quand on déplace la sonde de part et d'autre de cette position où le signal mesuré est minimal, on constate d'ailleurs un décalage de la sinusoïde observée sur l'écran de l'oscilloscope, qui prouve le déphasage de $180^{\circ}$ entre (2) et (3). Incidence et fréquence étant ainsi fixées, on déplace la sonde parallèlement à $\mathrm{O} x$ de façon à

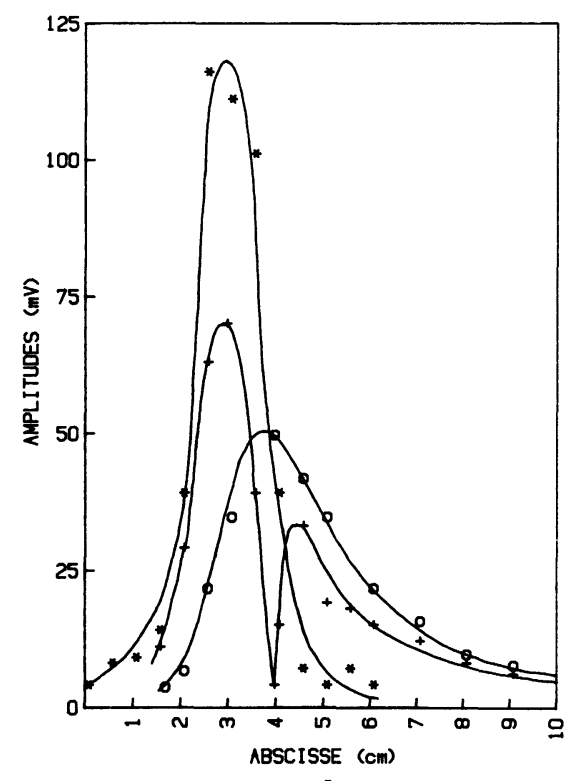

étudier successivement la répartition de l'amplitude le long du guide : (1) dans le faisceau retournant au milieu I (Fig. 3a), (2) dans le faisceau rayonné vers le milieu II (Fig. 3b), la sonde étant placée de l'autre côté de la plaque par rapport à l'émetteur et les axes des deux porte-traducteur étant parallèles, (3) dans le faisceau incident, l'exploration étant faite après avoir ôté la plaque (Fig. 3c).

Les amplitudes sont mesurées en $\mathrm{mV}$ crête à crête. Le bruit de fond est de l'ordre de $1,5 \mathrm{mV}$. Les figures $4 \mathrm{a}$ et $4 \mathrm{~b}$ donnent la répartition des amplitudes, dans les cas respectivement du mode $\mathrm{A}_{1}\left(i=15^{\circ}, f=3,45 \mathrm{MHz}\right)$ et du mode $\mathrm{S}_{0}\left(i=25^{\circ}, f=2,95 \mathrm{MHz}\right)$; on remarque que les pertes par rayonnement sont nettement plus fortes dans le second cas mais, dans les deux cas, les ondes retournant aux milieux I et II sont très voisines en dehors du faisceau incident et on observe bien un

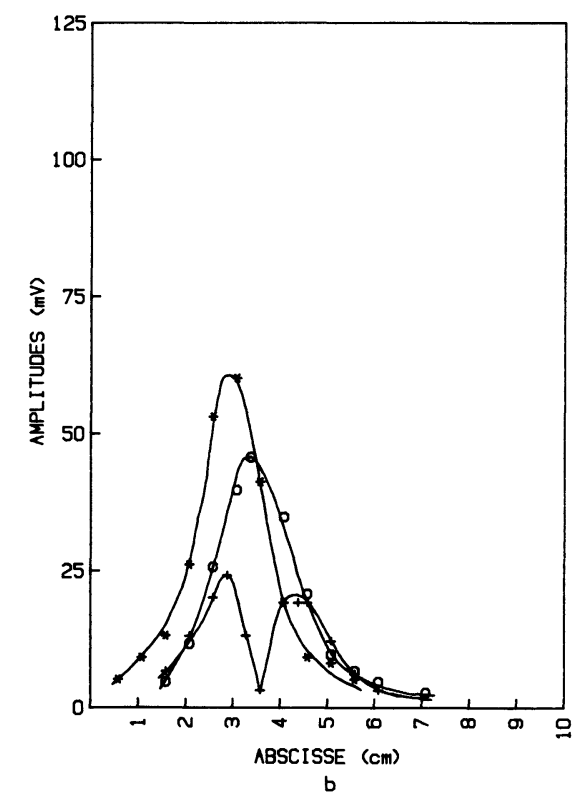

Fig. 4. - Répartition des amplitudes mesurées le long d'une plaque dans le faisceau incident (*), dans le faisceau retournant au milieu I (+) et dans le faisceau retournant au milieu II (O) : a) mode $\left.\mathrm{A}_{1}, i=15^{\circ}, f=3,45 \mathrm{MHz}, \mathrm{b}\right)$ mode $\mathrm{S}_{0}, i=25^{\circ}$, $f=2,95 \mathrm{MHz}$.

[Distribution of the measured amplitudes along a plate in the incident beam (*), in the beam to medium $I(+)$ and in the beam to medium II (O) : a) $\mathrm{A}_{1}$ mode, $i=15^{\circ}, f=3.45 \mathrm{MHz}$, b) $\mathrm{S}_{0}$ mode, $i=25^{\circ}, f=2.95 \mathrm{MHz}$.] 
point où l'onde retournant au milieu I s'annule quasiment. Pour des angles d'incidence inférieurs à $28^{\circ}$, il est difficile d'assurer qu'il n'y a pas de participation d'ondes longitudinales ou transversales, mais celle-ci devient vite négligeable dès qu'on s'éloigne du faisceau incident.

Si on utilise les équations (2) et (4) pour représenter les courbes des figures 4 , on peut en déduire la valeur du coefficient $r$, en calculant $\lambda$ à partir de $i$ et de $f$ et en faisant les hypothèses suivantes : (1) la distribution de l'amplitude dans le faisceau incident est gaussienne et sa largeur, mesurée à $\pm 1,5$ écarttype, est égale au diamètre de la pastille piézoélectrique de l'émetteur, divisé par cos $i\left(^{2}\right)$, (2) $k^{\prime} / k$ est voisin du rapport des impédances dans l'acier et dans l'eau pour les ondes considérées $\left({ }^{3}\right),(3) \alpha \simeq 0$. C'est ainsi que, pour le mode $\mathrm{A}_{1}, \lambda=1,7 \mathrm{~mm}, k^{\prime} / k \simeq 30$ et on peut estimer que $r=0,96$ à $3,45 \mathrm{MHz}$ (Fig. 5a); pour le mode $\mathrm{S}_{0}, \lambda=1,2 \mathrm{~mm}, k^{\prime} / k \simeq 17$ et $r=0,93$ à 2,95 MHz (Fig. 5b).

$\left(^{2}\right)$ Cette hypothèse correspond à un bon ajustement des courbes expérimentales donnant $I$ sur les figures 4.

$\left({ }^{3}\right) \mathrm{Ce} \cdot$ rapport n'a d'influence que sur l'amplitude de l'onde guidée relativement aux amplitudes des autres ondes.

\section{Onde continue et guide tubulaire sans réflecteur.}

Considérons un guide tubulaire; ce peut être un film déposé à la surface d'un barreau pour réaliser un laser [12] ou bien un tore d'aluminium pour amplifier des ondes acoustiques [13]. Cette configuration présente l'intérêt de fermer le guide sur lui-même, ce qui permet en particulier de réaliser un résonateur fonctionnant en ondes progressives comme en ondes stationnaires [14].

Nous avons mis au point un " prisme courbe " qui permet de coupler un tel guide avec une source plane annulaire, disposée coaxialement avec le guide [15]; il s'agit en fait d'un miroir qui défléchit de $90^{\circ}$ le faisceau émis par la source vers le tube et lui donne une incidence dans des plans équatoriaux du tube telle que la synchronisation entre ondes incidentes et ondes guidées est obtenue, sur une longueur d'arc qui peut atteindre $360^{\circ}$. Ainsi, l'énergie incidente est pratiquement constante sur toute la longueur $s$ de couplage et celui-ci peut être prolongé indéfiniment si on a :

$$
s=2 \pi R=q \lambda,
$$

en désignant par $R$ le rayon du tube et par $q$ un entier. Dans ces conditions, le coefficient d'amplification $A$ peut atteindre sa valeur à saturation donnée par (7),

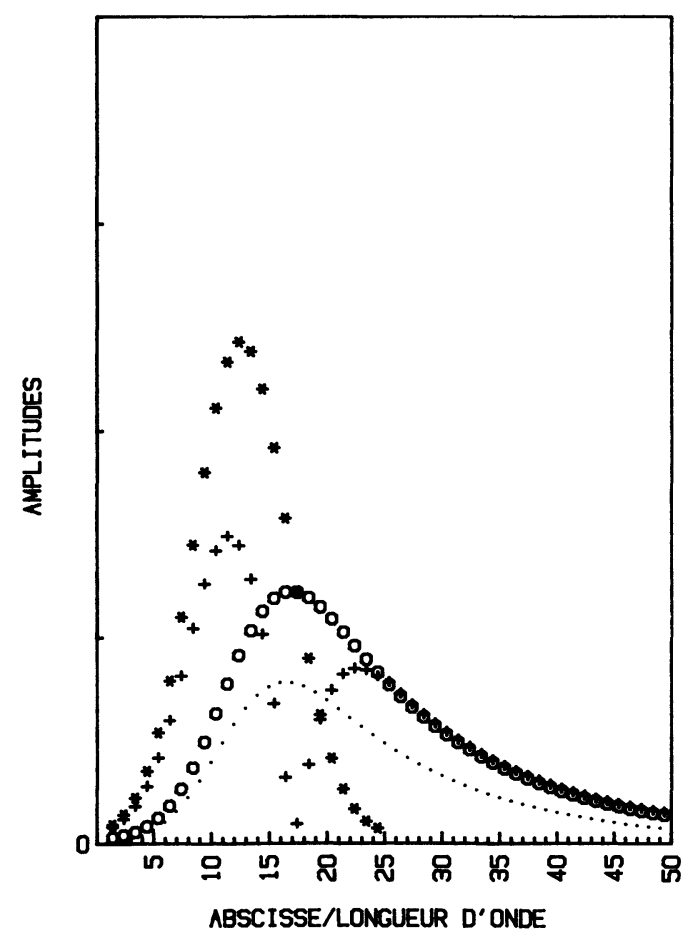

a

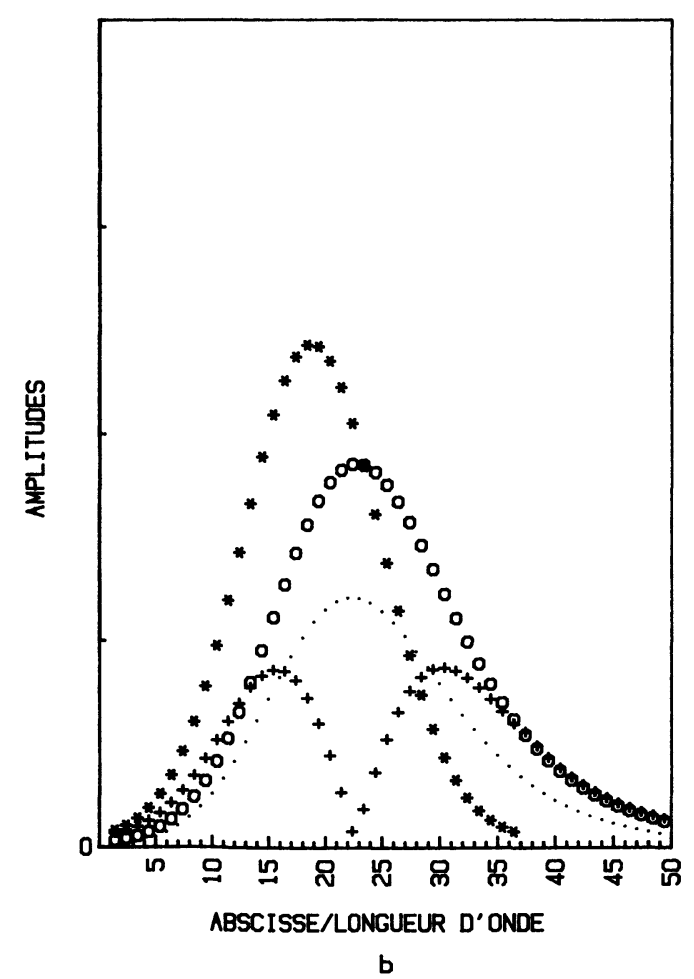

Fig. 5. - Répartition des amplitudes calculées le long d'une plaque dans le faisceau incident (*), dans le guide (.), dans le faisceau retournant au milieu I ( +) et dans le faisceau retournant au milieu II (O) : a) $\lambda=1,7 \mathrm{~mm}, \alpha=0, r=0,96, \mathrm{~b}) \lambda=$ $1,2 \mathrm{~mm}, \alpha=0, r=0,93$.

[Distribution of the calculated amplitudes along a plate in the incident beam (*), in the guide (.), in the beam to medium $\mathrm{I}(+)$ and in the beam to medium II (O) : a) $\lambda=1.7 \mathrm{~mm}, \alpha=0, r=0.96, \mathrm{~b}) \lambda=1.2 \mathrm{~mm}, \alpha=0, r=0.93$.] 
ce qui n'est guère possible avec un guide plat; or cette valeur à saturation devient très importante lorsque $r$ est voisin de 1.

La vérification expérimentale du modèle théorique est plus difficile qu'avec un guide plat. On peut cependant concevoir un dispositif de mesure, dont le principe est donné sur la figure 6. La sonde est déplacée en azimut de façon que son axe reste tangent à un cercle de rayon $R$ sin $i$; on mesure ainsi l'amplitude rayonnée en tout point de la face interne du guide, qui est proportionnelle à l'amplitude de l'onde guidée au niveau de ce point. Il faut néanmoins tenir compte des éventuelles ondes longitudinales ou transversales transmises par le tube.

\section{Train d'onde et guide plat avec réflecteur.}

Les ultrasons sont souvent utilisés pour détecter la présence d'obstacles; c'est le cas par exemple en contrôle non destructif [8] et l'émission se fait alors par impulsions, avec une fréquence de récurrence de l'ordre du $\mathrm{kHz}$ Quand ces impulsions sont utilisées pour engendrer des ondes guidées, elles doivent être suffisamment longues pour que l'énergie guidée ait le temps de croître. Pour simplifier, on considérera le cas d'un faisceau incident rectangulaire; on se rappelle en effet que l'énergie guidée croît alors linéairement avec la durée du couplage, au début de celui-ci ( $\$ 2.1$ ). Plutôt que des impulsions brèves, il est donc préférable d'utiliser des trains d'onde, qui permettent de plus un meilleur accord fréquentiel avec le mode à guider. On peut exprimer la durée du train d'onde incident en nombre $m$ de périodes; la longueur du train guidé sera ainsi $m \lambda$ et, pour tirer tout le profit de la longueur $s$ du guide le long de laquelle la condition de synchronisation entre source et guide est vérifiée, il faut choisir :

$$
m=s / \lambda \text {. }
$$

Soit une plaque plane comportant un réflecteur (Fig. 7). La grandeur d'un tel réflecteur est généralement déterminée à partir de l'amplitude de l'écho obtenu par réflexion d'ondes de Lamb [2]; on va donc chercher quels sont les paramètres affectant cette amplitude. Soit un train d'onde incident de durée $m$, couplé avec la plaque sur une longueur $s=\mathrm{AB}=m \lambda$, l'amplitude du train d'onde étant supposée constante et égale à $I$ de $\mathrm{A}$ à $\mathrm{B}$. Le train d'onde guidé aura son amplitude maximale en tête et, dans la mesure où le coefficient de réflexion $r$ est élevé, on n'observera pas de saturation et l'énergie du train d'onde croîtra de façon linéaire jusqu'à atteindre la valeur $k m\left(1-r^{2}\right) I^{2}$ au niveau de B. Soit $\rho$ le coefficient de réflexion du réflecteur $\left({ }^{4}\right)$, situé dans la plaque à une distance $d=\mathrm{BC}$. Compte tenu de l'atténuation entre B et $\mathrm{C}$

$\left.{ }^{4}\right)$ Pour un petit réflecteur, il s'agit plutôt d'une rétrodiffusion et $\rho$ est proportionnel à l'aire du réflecteur [8].

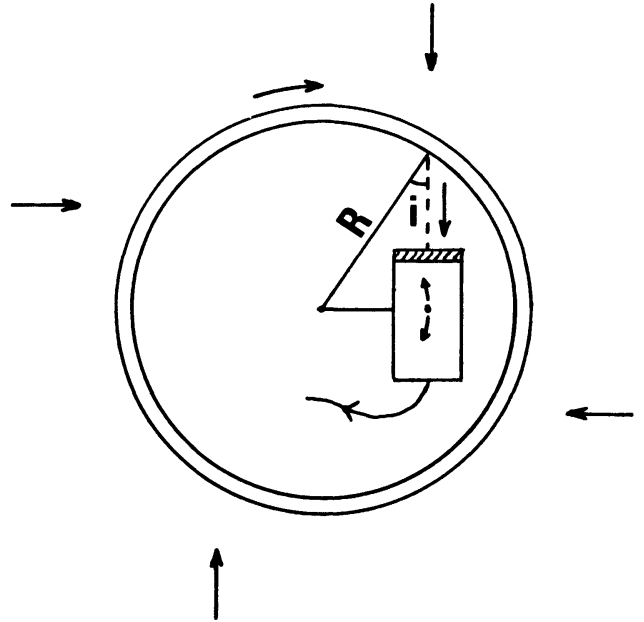

Fig. 6. - Principe de la mesure de l'amplitude du faisceau rayonné par la face interne d'un tube.

[Principle of the amplitude measurement of the beam radiated by the internal face of a tube.]

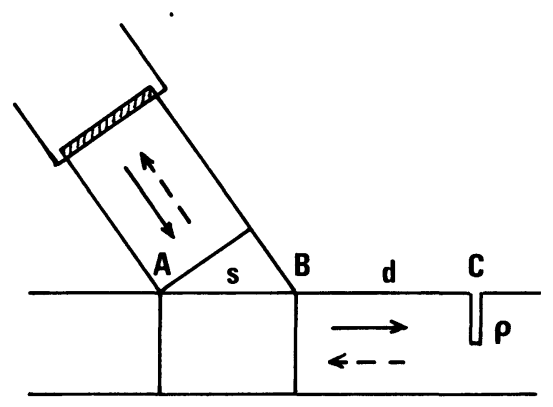

Fig. 7. - Principe de la mesure d'un écho en ondes de Lamb dans une plaque.

[Principle of the echo measurement with Lamb waves in a plate.]

puis entre $C$ et $B$, donnée par (10), l'énergie du train d'onde, de retour en $B$, ne sera plus que

$$
k m\left(1-r^{2}\right) I^{2} \rho^{2} \exp \times(-2 \beta d),
$$

ce qui correspond à une amplitude

$$
\left[\left(k / k^{\prime}\right) m\left(1-r^{2}\right)\right]^{1 / 2} \rho I \exp (-\beta d) .
$$

Le rapport entre l'amplitude maximale de l'écho rayonné en B vers la source, qui fonctionne maintenant comme récepteur, et l'amplitude du train d'onde qui a été émis par la source et incident en B, est donc :

$$
\begin{aligned}
E=t\left[\left(k / k^{\prime}\right) m(1-\right. & \left.\left.r^{2}\right)\right\rceil^{1 / 2} \rho I \exp (-\beta d) / I= \\
& =m^{1 / 2} \rho\left(1-r^{2}\right) \exp (-\beta d) .
\end{aligned}
$$

On voit que ce rapport $E$ croît avec la longueur de couplage, avec la réflectivité du réflecteur et décroît avec l'éloignement de celui-ci, ce qui était attendu; il croît aussi en principe quand le coefficient de réflexion 
décroît mais la relation (13) n'est valable que pour des valeurs de $r$ élevées, ce qui correspond d'ailleurs au cas habituel des contrôles.

\section{Train d'onde et guide tubulaire avec réflecteur.}

L'existence d'ondes ultrasonores circonférentielles, pouvant circuler dans un tube ou bien à la surface d'un cylindre, a été mise en évidence depuis longtemps sous forme d'une succession d'échos équidistants, avec un intervalle correspondant au temps nécessaire pour que l'onde effectue une révolution. On a d'abord observé des systèmes stationnaires, par rétrodiffusion d'une impulsion dirigée sur un objet cylindrique éloigné [16-19] : deux ondes circonférentielles de sens contraires sont ainsi engendrées et interfèrent; l'influence d'un réflecteur dans le guide a également été étudiée [20]. Puis des ondes progressives ont été observées, en utilisant un émetteur et un récepteur séparés, orientés selon une incidence correspondant à un angle critique de Rayleigh ou de Lamb [21]. Nous avons également obtenu des échos multiples dans des tubes avec des ondes progressives circonférentielles (ondes de Lamb), mais en échographie et en présence d'un réflecteur dans le tube [15], au moyen d'un " prisme courbe" $(\S 3)$. Nous avons observé à cette occasion que le premier écho n'était pas toujours le plus intense; c'est ce phénomène que nous allons chercher à expliquer $\left({ }^{5}\right)$.

Rappelons les conditions expérimentales. Un train d'onde sinusoïdal, de fréquence $4,5 \mathrm{MHz}$, de durée $2,5 \mu$ s et de tension $\pm 12 \mathrm{~V}$ (Fig. 8), fourni par un appareil CGR du type ER 30, est utilisé pour exciter un traducteur piézoélectrique plan annulaire de diamètres $20 \times 30 \mathrm{~mm}$, de fréquence $4 \mathrm{MHz}$ avec un amortissement moyen, fabriqué par NDC; la fréquence de récurrence est de l'ordre du $\mathrm{kHz}$. Le guide est constitué par un tube en zircaloy plein d'air, de diamètre extérieur $9,5 \mathrm{~mm}$ et d'épaisseur $0,6 \mathrm{~mm}$, comportant un réflecteur; celui-ci est une fente électroérodée, de longueur $1,5 \mathrm{~mm}$ et de profondeur $0,05 \mathrm{~mm}$, orientée parallèlement à l'axe du tube. Le couplage est assuré dans l'eau, par un miroir hélicoïdal (Eq. (3.35) de [8]) engendré par la tangente à une hélice de rayon $1,4 \mathrm{~mm}$; il correspond donc à un angle d'incidence $i=17,1^{\circ}$ (Eq. (3.34) de [8]) et, compte tenu des diamètres de la couronne émettrice, le train d'onde est couplé sur une largeur de $5 \mathrm{~mm}$ et sur une longueur d'arc de 206 (Eqs. (3.36) et (3.37) de [8]). Dans ces conditions, on observe une succession d'échos (Fig. 9), dont l'amplitude est maximale lorsque le réflecteur est approximativement situé à la fin de l'arc couplé; la distance entre échos est de 9,3 $\mu$ s. Comme la circonférence est égale à $29,9 \mathrm{~mm}$, on en déduit une vitesse

$\left({ }^{5}\right)$ Il est possible de visualiser simplement la propagation d'ondes circonférentielles, au moyen d'un ressort à boudin dont on sonde les extrémités et que l'on suspend horizontalement ; le réflecteur est alors formé par une masselotte [10].

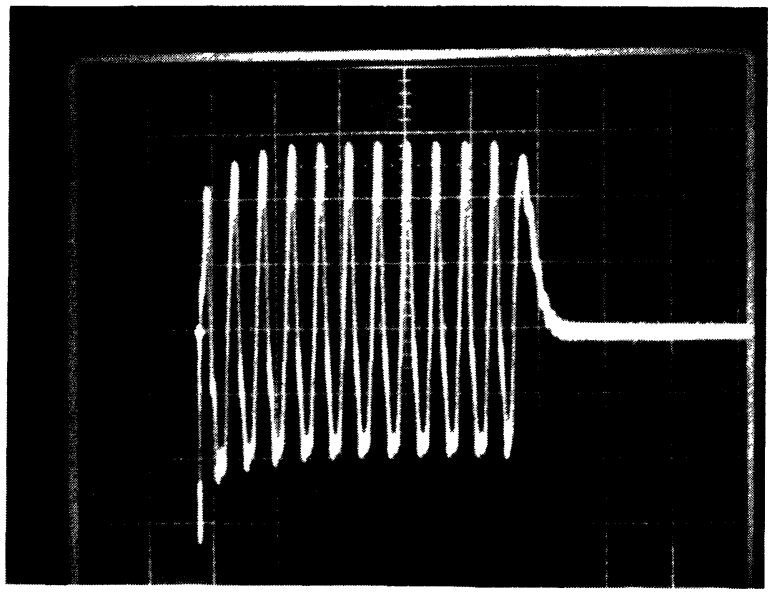

Fig. 8. - Train d'onde émis $(0,5 \mu \mathrm{s} /$ div., $5 \mathrm{~V} /$ div. $)$.

[Transmitted pulse $(0.5 \mu \mathrm{s} /$ div., $5 \mathrm{~V} /$ div. $)$.]

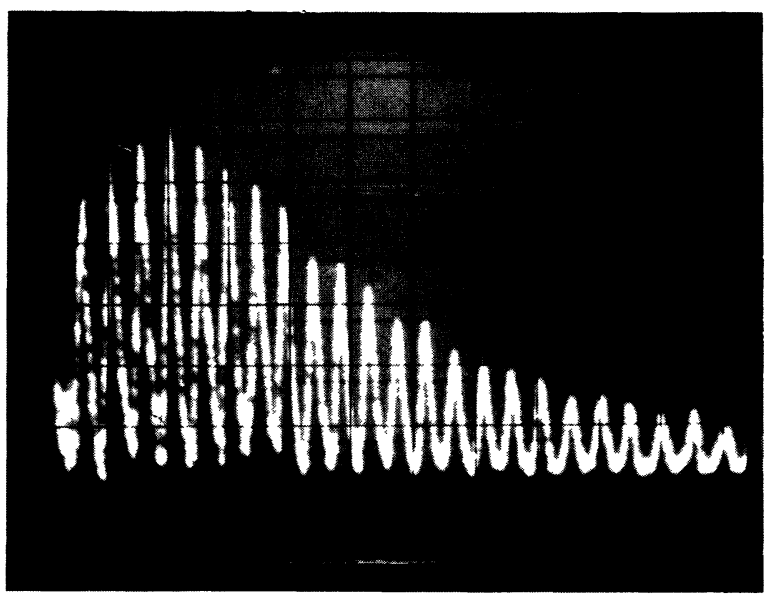

Fig. 9. - Echo multiple observé dans un tube comportant un réflecteur $(17 \mu \mathrm{s} /$ div. $)$.

[Multiple echo in a tube with a reflector $(17 \mu \mathrm{s} / \mathrm{div}$.$) .]$

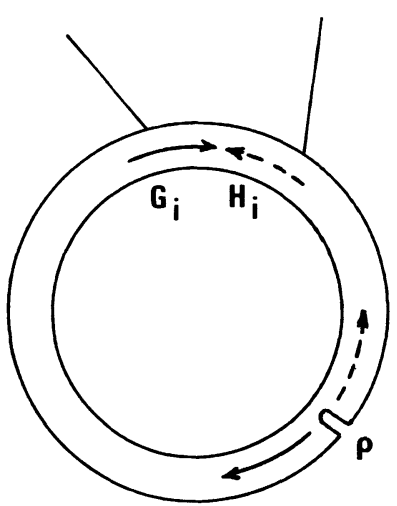

Fig. 10. - Répartition des amplitudes dans un tube comportant un réflecteur.

[Amplitude distribution in a tube with a reflector.] 
de groupe de l'ordre de $3600 \mathrm{~m} / \mathrm{s}$; par ailleurs, l'angle d'incidence dans l'eau correspond à une vitesse de phase de l'ordre de $5100 \mathrm{~m} / \mathrm{s}$. Compte tenu du produit fréquence $\times$ épaisseur, il doit s'agir du mode $S_{1}$ [11] On observe enfin que l'amplitude maximale est obtenue pour le quatrième écho (Fig. 9).

Considérons un train d'onde incident sur un tube (Fig. 10), sous une incidence permettant d'engendrer un train d'onde guidé; soit $G$ l'amplitude de ce train au moment où il rencontre le réflecteur, de coefficient de réflexion égal à $\rho\left({ }^{4}\right)$. La première réflexion sur le réflecteur conduit à deux trains guidés : (1) un train d'amplitude $G_{1}=(1-\rho) G$, tournant dans le même sens que le train guidé initial, (2) un train d'amplitude $H_{1}=\rho G$, tournant en sens inverse. Après avoir effectué un tour et s'être ainsi atténué d'un coefficient $\exp (-2 \pi R \beta), \beta$ étant donné par la relation (10), chaque train d'onde va subir à nouveau une réflexion de coefficient $\rho$ et une transmission de coefficient $(1-\rho)$, en supposant que le réflecteur est parfaitement symétrique pour les deux sens de propagation. Si on suppose en plus que le guide est peu dispersif, les trains y circulent sans modification importante de leur forme; ainsi les ondes $G_{i}$ et $H_{i}$ se retrouvent en phase à chaque passage devant l'émetteur, qui fonctionne maintenant en récepteur, et leurs amplitudes peuvent s'ajouter. La mise en équation, effectuée par Pouchin [10], conduit à :

$$
\begin{aligned}
& G_{n} \exp (2 \pi R \beta)=(1-\rho) G_{n-1}+\rho H_{n-1}, \\
& H_{n} \exp (2 \pi R \beta)=\rho G_{n-1}+(1-\rho) H_{n-1}
\end{aligned}
$$

et la résolution du système donne l'amplitude $\mathrm{du}$ $n$-ième écho :

$$
H_{n} / G=\exp (-2 \pi(n-1) R \beta)\left[1-(1-2 \rho)^{n}\right] / 2 ;
$$

la courbe d'équation $y=H(n) / G$ représente l'enveloppe de l'écho multiple.

On a tracé cette courbe enveloppe pour diverses valeurs de $\beta$ (Fig. 11); on a pris $2 R=9,5 \mathrm{~mm}$ et on a considéré que $\rho$ était égal au rapport de la section du réflecteur $\left(1,5 \times 0,05=0,075 \mathrm{~mm}^{2}\right)$ sur celle du faisceau guidé $\left(5 \times 0,6=3 \mathrm{~mm}^{2}\right)$, soit $\rho=0,025$. La courbe correspondant à $\beta=6 \mathrm{~m}^{-1}$ coïncide assez bien avec l'allure de l'écho multiple observé sur la figure 9; lą relation (10) permet d'en déduire un coefficient $r$ très voisin de 1 . Plus généralement, on peut remarquer que la première partie de la courbe est principalement déterminée par $\rho$ et renseigne donc sur la nature du réflecteur, alors que la seconde est principalement déterminée par $\beta$.

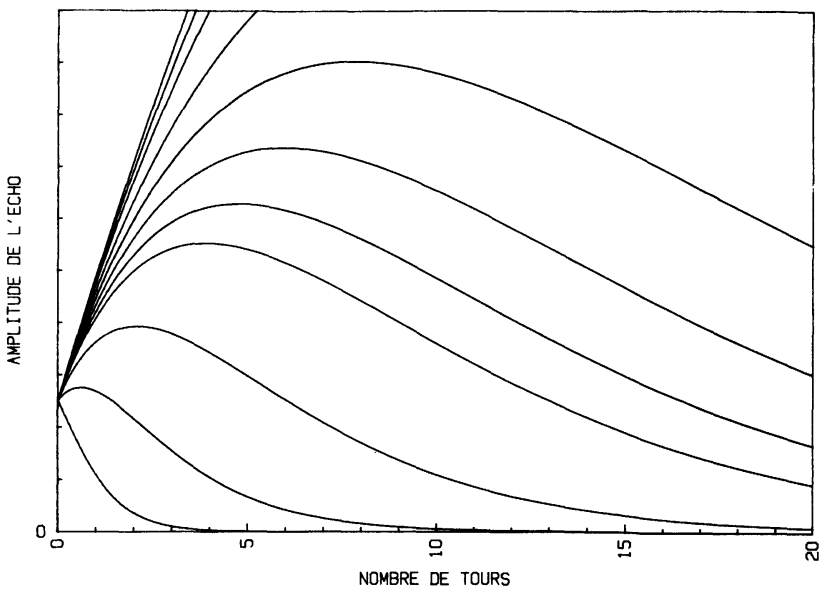

Fig. 11. - Enveloppe de l'écho multiple dans un tube de diamètre $9,5 \mathrm{~mm}$ pour $\rho=0,025$ et $\beta=0,1 ; 0,5 ; 1 ; 2$; $3 ; 4 ; 5 ; 6 ; 10 ; 20$ et $50 \mathrm{~m}^{-1}$ (de haut en bas).

[Envelope of the multiple echo in a tube of diameter $9.5 \mathrm{~mm}$, with $\rho=0.025$ and $\beta=0.1 ; 0.5 ; 1 ; 2 ; 3 ; 4 ; 5 ; 6 ; 10 ; 20$ and $50 \mathrm{~m}^{-1}$ (from top to bottom).]

\section{Conclusions}

La démarche, qui a été suivie ici pour étudier la transmission entre une onde libre et une onde guidée, était très pragmatique. Elle n'avait pas en effet pour but de déterminer les intensités des divers faisceaux à partir des propriétés des milieux, ce qui a déjà été étudié par ailleurs. Il s'agissait seulement de trouver un modèle simple pour calculer les intensités des divers faisceaux, et de comparer ensuite ce modèle à la réalité.

Ce modèle est donné par une équation différentielle et nous avons montré qu'il permettait de représenter correctement le profil des amplitudes dans les faisceaux ultrasonores environnants une plaque plane guidant des ondes de Lamb; nous avons remarqué à cette occasion l'intérêt d'un guide tubulaire pour obtenir une énergie guidée maximale. $\mathrm{Ce}$ modèle a ensuite été appliqué à l'échographie; nous avons montré l'intérêt d'utiliser des trains d'onde pour engendrer des ondes de Lamb et nous avons expliqué l'origine de l'écho multiple, que nous avions observé dans un tube comportant un réflecteur.

\section{Remerciements.}

Nous tenons à remercier MM. Pouchin et Sladkoff pour leur contribution à cette étude. 


\section{Bibliographie}

[1] Kogelnik, H., Coupling of optical devices and waveguides, dans Photonics, Balkanski, M. et Lallemand, P., éditeurs (Gauthier-Villars, Paris) 1973.

[2] Viktorov, I. A., Rayleigh and Lamb waves (Plenum Press, New York) 1970.

[3] TIEN, P. K. et UlRICH, R., Theory of prism-film coupler and thin-film light guides, J. Opt. Soc. Am. 60 (1970) 1325.

[4] Neubauer, W. G., Ultrasonic reflection of a bounded beam at Rayleigh and critical angles for a plane liquid-solid interface, J. Appl. Phys. 44 (1973) 48.

[5] ULRICH, R., Theory of the prism-film coupler by planewave analysis, J. Opt. Soc. Am. 60 (1970) 1337.

[6] BerTONI, H. L. et TAMIR, T., Unified theory of Rayleighangle phenomena for acoustics beams at liquidsolid interfaces, Appl. Phys. 2 (1973) 157.

[7] Fiorito, R., Madigosky, W. et Uberall, H., Resonance theory of acoustic waves interacting with an elastic plate, J. Acoust. Soc. Am. 66 (1979) 1857.

[8] Perdijon, J., L'échographie (Dunod, Paris) 1981.

[9] Voir par exemple, Florent, P., LAUton, G. et LAUTON, M., Equations et systèmes différentiels (Vuibert, Paris) 1978.

[10] Pouchin, B., La résonance annulaire des ondes de Lamb lors du contrôle des tubes minces, projet de fin d'études soutenu le 27 juin 1983, Ecole Nationale Supérieure d'Ingénieurs Electriciens de Grenoble.

[11] Worlton, D. C., Lamb waves at ultrasonic frequencies, rapport Hanford HW-60662 (1959).

[12] Weber, H. P. et Ulrich, R., A thin-film ring laser, Appl. Phys. Lett. 19 (1971) 38.
[13] Ostrovskil, L. A., Papilova, I. A. et Sutin, A. M., Parametric generation and amplification of acoustic waves in a solid annular resonator, Sov. Phys. Tech. Phys. 18 (1974) 1403.

[14] TISChER, F. J., Resonance properties of ring circuits, IRE Trans. Micro-wave Theory Tech. MTT 5 (1957) 51.

[15] Perdijon, J., Optique de couplage encerclant, Revue Phys. Appl. 18 (1983) 129.

[16] Diercks, K. J., Goldsberry, T. G. et Horton, C. W., Circumferential waves in thin-walled air-filled cylinders in water, J. Acoust. Soc. Am. 35 (1963) 59.

[17] Doolittle, R. D., Uberall, H. et Ugincius, P., Sound scattering by elastic cylinders, J. Acoust. Soc. Am. 43 (1968) 1.

[18] MAZE, G. et Ripoche, J., Méthode d'isolement et d'identification des résonances de cylindres et de tubes soumis à une onde acoustique plane dans l'eau, Revue Phys. Appl. 18 (1983) 319.

[19] FeKiH, M. et Quentin, G., Scattering of short ultrasonic pulses by thin cylindrical shells; generation of guided waves inside the shell, Phys. Lett. 96A (1983) 379.

[20] GolDSBerRy, T. G., Reflection of circumferential waves from a slit in a thin-walled cylinder, J. Acoust. Soc. Am. 42 (1967) 1298.

[21] Bunney, R. E., Goodman, R. R. et Marshall, S. W., Rayleigh and Lamb waves on cylinders, J. Acoust. Soc. Am. 46 (1969) 1223. 